\title{
El marketing educativo como estrategia para la satisfacción de alumnos universitarios
}

\section{Educational Marketing as a Strategy for the Satisfaction of University Students}

\author{
Liliana D.J. Gordillo*
}

Universidad Politécnica de Tulacingo, México

ORCID: https://orcid.org/0000-0001-9982-7283

\section{Benedicta M. Domínguez}

Universidad Politécnica de Tulacingo, México

ORCID: https://orcid.org/0000-0002-5739-8940

\section{Claudia Vega}

Universidad Politécnica de Tulacingo, México

ORCID: https://orcid.org/0000-0001-5204-5081

Adriana De la Cruz

Universidad Católica de Temuco, Chile

ORCID: https://orcid.org/0000-0001-7155-5204

Mario Angeles 10

Universidad Politécnica de Tulancingo, México

ORCID: https://orcid.org/0000-0001-5852-6222

Recibido 02-01-20 Revisado 15-02-20 Aprobado 30-03-20 En línea 07-04-20

*Correspondencia

Email: liliana.gordillo@upt.edu.mx
Citar como:

Gordillo, L. J. D., Domínguez, B. M., Vega, C., De la Cruz, A., \& Angeles, M. (2020). El marketing educativo como estrategia para la satisfacción de alumnos universitarios. Propósitos y Representaciones, 8(SPE1), e499. http://dx.doi.org/10.20511/pyr2020.v8nSPE1.499 


\section{Resumen}

Las instituciones de educación superior se enfocan en la captación de alumnado, así como de su fidelización a la institución, esto se logra mediante la satisfacción en el estudiante. El objetivo de esta investigación fue analizar el marketing educativo como estrategia de satisfacción de los alumnos de la Universidad Politécnica de Francisco I, Madero (UPFIN). El estudio tiene un enfoque cuantitativo de tipo descriptiva, se aplicó a una muestra de 361 estudiantes de la UPFIN y algunos resultados fueron que para lograr la satisfacción del educando intervienen variables como: los vídeos de enseñanza, servicio de biblioteca digital, vinculación con microempresas, clases extras para los que reprueban, horas extras de enseñanza de segundo idioma, orientación psicológica y social, guía de estudios para presentar exámenes, salones y laboratorios en buenas condiciones, plan acorde a la carrera, congresos y simposium en diferentes carreras, involucramiento de estudiantes en proyectos de investigación, trato amable de los administrativos, becas para pasajes de alumnos, demostraciones y capacitaciones con microempresarios y público en general de lo que se realiza en la universidad.

Palabras clave: Marketing educativo; Satisfacción; Estrategia

\section{Summary}

In a globalized and competitive world, the need for the Higher Education Institutions to have a competitive advantage and exceed the expectations of the learners becomes more and more imperative, although the fundamental part of the Institutions is also true in addition to capturing enrollment is to retain it. and to retain it, and to achieve the above, it requires student satisfaction, so the objective of this research was to propose educational marketing as a strategy for student satisfaction at the Polytechnic University of Francisco I, Madero (UPFIN). The study has a quantitative approach of a descriptive type, it was applied to a sample of 361 UPFIN students and some results were that in order to achieve student satisfaction, variables such as: teaching videos, digital library service, linkage with microenterprises, extra classes for those who fail, overtime teaching second language, psychological and social guidance, study guide to present exams, classrooms and laboratories in good condition, plan according to the race, congresses and symposium in different careers, student involvement in research projects, friendly treatment of administrative staff, scholarships for student tickets, demonstrations and training with microentrepreneurs and the general public of what is done in the university.

Keywords: Educational Marketing; Satisfaction; Strategy.

\section{Introducción}

En la actualidad las Instituciones de Educación Superior juegan un papel importante para el desarrollo económico. Aunado a esto, en el Plan Estatal de Desarrollo (2017-2022). dentro del Programa Institucional de Desarrollo de la Universidad Politécnica de Francisco I. Madero (UPEFIM), señala como retos las grandes transformaciones de las Instituciones y la sociedad en general y deberán desarrollarse en un ambiente de alta competitividad. Partiendo de esa idea menciona que la calidad, la tecnología, el servicio y los recursos altamente preparados se convierten en factores críticos de éxito (Gobierno del Estado de Hidalgo, 2016).

De acuerdo con Salinas y Martínez (2007), la satisfacción del alumno en los estudios universitarios ha cobrado vital importancia para las instituciones, pues de ella depende su supervivencia. Sólo con la satisfacción de los alumnos se podrá alcanzar el éxito una valoración positiva boca a boca. En este sentido, es extremamente importante encontrar formas fiables de medir la satisfacción del alumno en la enseñanza universitaria, permitiendo así a las instituciones 
de enseñanza conocer su realidad, compararla con la de los otros competidores y analizarla a lo largo del tiempo.

El término satisfacción se encuentra estrechamente relacionado a la calidad, explicada como el resultado de comparar las expectativas con la percepción del servicio recibido. Esto significa que la medida de la calidad está en la satisfacción de las personas y en el valor de lo que reciben. La efectividad, como relación entre las necesidades sentidas y los resultados percibidos es la única escala válida para medir la calidad. El éxito de los programas o de las organizaciones radica esencialmente en responder a la maximización de esta proporción (Lago, López, Municio, Ospina \& Vergara 2013, p. 37).

A través del contexto universitario donde se ve el aumento de las universidades tanto públicas como privadas, las instituciones de educación superior deben generar ventaja competitiva y por ende lograr satisfacción en los educando, es así como la satisfacción a los clientes se ha convertido en el eje central mercadológico; las organizaciones perciben el valor de un cliente satisfecho en términos de actitudes positivas hacia la marca, el boca en boca efectivo, las compras repetidas y la lealtad (Spreng, MacKenzie y Olshavsky, 1996). La satisfacción del alumnado generalmente es aceptada como una actitud a corto plazo que resulta de una evaluación de la experiencia educativa del colegial (Elliott y Healy, 2001), y repercute positivamente cuando el rendimiento real cumple o supera las expectativas de los estudiantes.

\section{Marketing Educativo}

De acuerdo con Manes (2004), las instituciones educativas están inmersas en un proceso de cambio que quizá sea demasiado veloz para sus tiempos institucionales. Si embargo, es necesario que las personas a cargo asuman la real utilidad de nuevas herramientas que, como el marketing, ayudan a mejorar la gestión institucional y coadyuvan al proceso de cambio.

Desde la nueva perspectiva institucional, las escuelas deben reconocer que:

a) El marketing educativo es complementario de una gestión administrativa eficiente.

b) Hay limitaciones del uso del marketing por parte de los establecimientos educativos, sobre todo en la educación formal.

c) Existe un conocimiento limitado del marketing por parte de las instituciones educativas y su correcta aplicación.

d) Hay una diferencia entre el marketing mínimo, el marketing equilibrado y el marketing comercial.

e) En las instituciones educativas existen diferentes estructuras culturales, historia y propósitos.

f) Es necesario evaluar de las decisiones administrativas y organizacionales dentro del marco de la ética.

g) Es necesario que los directivos comprendan la necesidad de capacitación en temas de marketing y management (gerenciamiento) educativo.

h) Es necesario entender que hoy la institución educativa debe incorporar el marketing como proceso y designar un responsable del área al servicio de la comunidad.

Larios-Gómez (2014), menciona que la mercadotecnia educativa es la herramienta estratégica de la gestión, que ejecutan las instituciones de educación (de todos los niveles o grados escolares) y que permite satisfacer necesidades de desarrollo personal para la adquisición de nuevo conocimiento, a través de la comercialización (intercambio y venta) de un servicio, de forma directa o indirecta (presencial o virtual).

Así mismo el marketing educativo es el proceso de investigación de las necesidades sociales, para desarrollar servicios educativos tendientes a satisfacerlas, acordes a su valor 
percibido, distribuidos en tiempo y lugar y éticamente promocionados para generar bienestar entre individuos y organizaciones (Manes, 2004).

Si bien es cierto el marketing educativo ha atravesado por una serie de complicaciones por lo que muchas instituciones no lo aplican al 100\%. De acuerdo con Zapata (2012) la mercadotecnia educativa, es un tópico poco abordado para algunas instituciones, sin embargo, es conveniente colocarlo al servicio del proceso de crecimiento de las instituciones de educación superior. Es así como la importancia del marketing educativo se incrementa de manera significativa en el sector educativo en todos sus niveles.

El marketing educativo, es el proceso de investigación de las necesidades sociales, para desarrollar servicios educativos tendientes a satisfacerlas, acordes a su valor percibido, distribuido en tiempo y lugar, y éticamente promocionados para generar bienestar entre individuos y organizaciones (Manes, 2004, p.15) el marketing educativo es la satisfacción de las necesidades individuales y sociales que permitan servicios educativos de calidad en el tiempo y lugar determinado, creando valor para el bienestar de la sociedad.

Alvarado (2003), destaca que el marketing educativo es el cumplimiento de la acción comercial que orienta el flujo del servicio, desde las universidades hacia los consumidores del servicio para satisfacer necesidades. La calidad educativa es aquella en el que los resultados conjugan la calidad de promesa y calidad de demanda.

\section{Satisfacción estudiantil}

De acuerdo con Jiménez, Terriquez y Robles (2012), la satisfacción del estudiante refleja la eficiencia de los servicios académicos y administrativos. Importante saber que los estudiantes manifiesten su satisfacción con las unidades de aprendizaje, con las interacciones con su profesor y compañeros de clase, así como con las instalaciones y el equipamiento. Son los estudiantes los principales usuarios de los servicios universitarios, ellos son los que mejor pueden valorarla, y aunque pueden tener una visión parcial, sus opiniones sirven como indicador de mejoramiento de la gestión y el desarrollo de los programas académicos.

Garbanzo (2007), conceptualiza la satisfacción estudiantil como el bienestar que experimentan los alumnos por sentir cubiertas sus expectativas académicas como resultado de las actividades que realiza la institución para atender sus necesidades educativas. Los estudios sobre satisfacción estudiantil en las universidades como indicadores para evaluar la calidad educativa son particularmente importantes, porque la satisfacción estudiantil mejora el rendimiento académico, a su vez Sinclaire (2014) menciona, que el rendimiento académico es un requisito para el éxito en el aprendizaje. Si bien es cierto, las instituciones educativas, en esa búsqueda de oportunidades para mejorar, han venido identificando factores para evaluar la satisfacción estudiantil en sintonía con las tendencias en gestión de la calidad y excelencia en el desempeño, lograr conocer la dimensión de la satisfacción de los estudiantes con la institución a la cual concurren, permitirá identificar aspectos tanto positivos como negativos, siendo estos últimos fundamentales al momento de determinar estrategias de mejora de la educación. Es así como la satisfacción se convierte en la esencia de la mercadotecnia en la cual los alumnos estén satisfechos con el producto educativo recibido por tal motivo hay que ver si las expectativas que los estudiantes al ingresar a la institución se cumplieron al hacer uso de lo que se recibió (CadenaBadilla, Mejías, Vega \& Vázquez, 2015).

Hill, Brierley y MacDougall (2003), contemplan que un programa de medición de la satisfacción del cliente genera capacidades para: comprender cómo los clientes perciben su organización y si su desempeño satisface sus expectativas, identificar áreas prioritarias para mejoramiento, donde mejoras en desempeño producirán la más grande ganancia en satisfacción del cliente, emprender un análisis costo-beneficio para evaluar el impacto, localizar brechas de comprensión donde los colaboradores tienen interpretaciones equivocadas sobre las prioridades 
de sus clientes o sobre las competencias que pueden satisfacer las necesidades de los clientes, establecer metas para mejoramiento de servicios y hacer seguimiento del progreso en el índice de satisfacción, comparar el desempeño con el de otras organizaciones del mismo giro e incrementar los beneficios o la rentabilidad a través del mejoramiento de la lealtad y retención del cliente.

En cualquier organización, además de la captación de los clientes, es necesario la retención y la fidelización de estos, considerando que para que se logre lo anteriormente expuesto se debe de contar con la satisfacción total de los clientes, incluyendo estrategias que permitan la satisfacción de estos.

La satisfacción, es un estado cognitivo y emocional gratificante en el que se materializa la percepción del ser humano frente al cumplimiento de sus expectativas. Bajo este tenor radica la importancia que tiene la satisfacción del educando para tener éxito en la Institución (Sánchez, 2018).

\section{Estrategia}

Toda empresa pública o privada establecen estrategias para alcanzar los objetivos organizacionales, Para Chandler (2003), la estrategia es la determinación de las metas y objetivos de una empresa a largo plazo, las acciones a emprender y la asignación de recursos necesarios para el logro de dichas metas. Cabe destacar que el autor refiere a la estrategia a largo plazo ya que las empresas tienden a sobrevivir por la aplicación de las estrategias, así mismo involucra tiempo y aplicación de recursos financieros, tecnológicos, y humanos.

Según Carneiro (2010), la estrategia es la orientación en el actuar futuro, es el establecimiento de un fin, en un plazo estimado como aceptable hacia el cual orientar el rumbo empresarial. Cabe destacar la importancia de que se establezcan estrategias para lograr el éxito organizacional.

\section{Método}

Esta investigación tuvo un enfoque cuantitativo, que de acuerdo con Hernández, Fernández y Baptista (2014) tiene la característica de datos que se pueden medir y se establece un análisis estadístico. Se realizó un estudio descriptivo, que presenta el grado de satisfacción por el grupo objetivo que son los estudiantes de la Universidad Politécnica de Francisco I. Madero. El diseño de investigación fue no experimental ya que el fenómeno se realiza en su contexto natural sin que el investigador influya en las variables a medir, es transaccional ya que la recolección de datos se realizó en un tiempo determinado.

En la presente investigación se analizó la variable marketing educativo; en la que se evalúan diversos aspectos, como se observa que se explican en la Tabla 1. 
Tabla 1.

Conceptualización y operacionalización de variables

\begin{tabular}{|c|c|c|}
\hline Variable & $\begin{array}{l}\text { Definición } \\
\text { conceptual }\end{array}$ & Definición operacional \\
\hline $\begin{array}{l}\text { Producto } \\
\text { educativo }\end{array}$ & $\begin{array}{l}\text { Es todo lo que el servicio } \\
\text { ofrece la institución lo } \\
\text { cual incluye los planes y } \\
\text { programas de estudio e } \\
\text { instalaciones Espinoza } \\
(2010) \text {. }\end{array}$ & $\begin{array}{l}33 \text { ítems que miden el producto } \\
\text { educativo como: becas, área de } \\
\text { vinculación, asignaturas prácticas, } \\
\text { asesorías académicas, cursos de inglés, } \\
\text { guías de estudios. Plan de carrera adhoc, } \\
\text { actividades extracurriculares, } \\
\text { infraestructura adecuada, difusión de la } \\
\text { institución, orientación psicológica y } \\
\text { social, servicio al cliente, plan de } \\
\text { estudios con reconocimiento, } \\
\text { motivación constante, personal } \\
\text { capacitado, cursos de actualización, } \\
\text { inclusión de estudiantes en proyectos, } \\
\text { congresos en diferentes carreras, } \\
\text { biblioteca digital, servicio médico, } \\
\text { apoyos en gestión administrativas, } \\
\text { expectativas de alumnos, intercambios } \\
\text { académicos, transferencia tecnológica, } \\
\text { servicio de internet, programas de } \\
\text { tutorías, cafetería escolar, } \\
\text { estacionamientos.. Áreas verdes. }\end{array}$ \\
\hline
\end{tabular}

Fuente: Elaboración Propia

\section{Selección de muestra}

La muestra está formada por estudiantes mujeres y hombres que cursan el cuatrimestre mayoagosto 2019 en la UPFIM cuenta con una matrícula de 1581 estudiantes en total, dividido en los diferentes programas académicos que oferta la universidad tal como se muestra en la tabla 2.

Tabla 2

Oferta educativa UPFIM en diferentes programas académicos

\begin{tabular}{llc}
\hline Nivel & Programa Educativo & Matrícula \\
\hline Licenciatura & Ing. Agro Industrial & 144 \\
& Ing. Agro tecnología & 295 \\
& Ing. Sistemas computacionales & 263 \\
& Ing. Financiera & 275 \\
& Ing. Civil & 429 \\
& Ing. Diseño Industrial & 93 \\
& Ing. Energías & 66 \\
Maestría & Ciencias en desarrollo Agro tecnológico & 16 \\
Total & Sustentable & \\
\hline
\end{tabular}

Fuente: Servicios escolares UPFIM (2019).

Para determinar la muestra se tomó la fórmula estadística para poblaciones finitas con un intervalo de confianza de $95 \%$ y un error de estimación de 5\%, aplicando la siguiente ecuación.

$$
n=\frac{Z^{2}{ }^{*}{ }^{*} q^{*} N}{(N-1) E^{2}+p^{*} q}
$$


Con la aplicación de la fórmula dio un resultado de 361 participantes.

\section{Instrumentos de recolección de datos}

Para la recolección de los datos sobre las variables que se determinaron como se muestra en la tabla de conceptualización y operacionalización de variables, se utilizó un cuestionario estructurado, por afirmaciones.

El cuestionario fue conformado por 66 ítems, con dos diferentes tipos de escala Likert tal como se muestra a continuación:

1. Nada importante

2. Poco importante

3. Ni importante ni no importante

4. Importante

5. Muy importante

Mientras que para la evaluación de satisfacción se emplea:

1. Nada Satisfecho

2. Poco Satisfecho

3. Ni satisfecho ni insatisfecho

4. Satisfecho

5. Muy satisfecho

\section{Resultados}

Los resultados de la investigación se llevó a cabo con un mapa perceptual de satisfacción para ser más precisos de las frecuencias en las respuestas, se obtuvo con las variables de estudio para este caso, marketing educativo, importancia y satisfacción, este mapa perceptual está dando por cuatro cuadrantes el eje de las X y el eje de las Y, para el eje de las X se tiene plasmado la importancia que tiene el marketing educativo, y el eje de las Y la satisfacción del educando con el marketing educativo, por lo que se puede interpretar que mientras más cerca se ubiquen en el origen de las coordenadas mayor satisfacción e importancia con respecto a los indicadores del marketing educativo como se puede apreciar en la figura 1. 


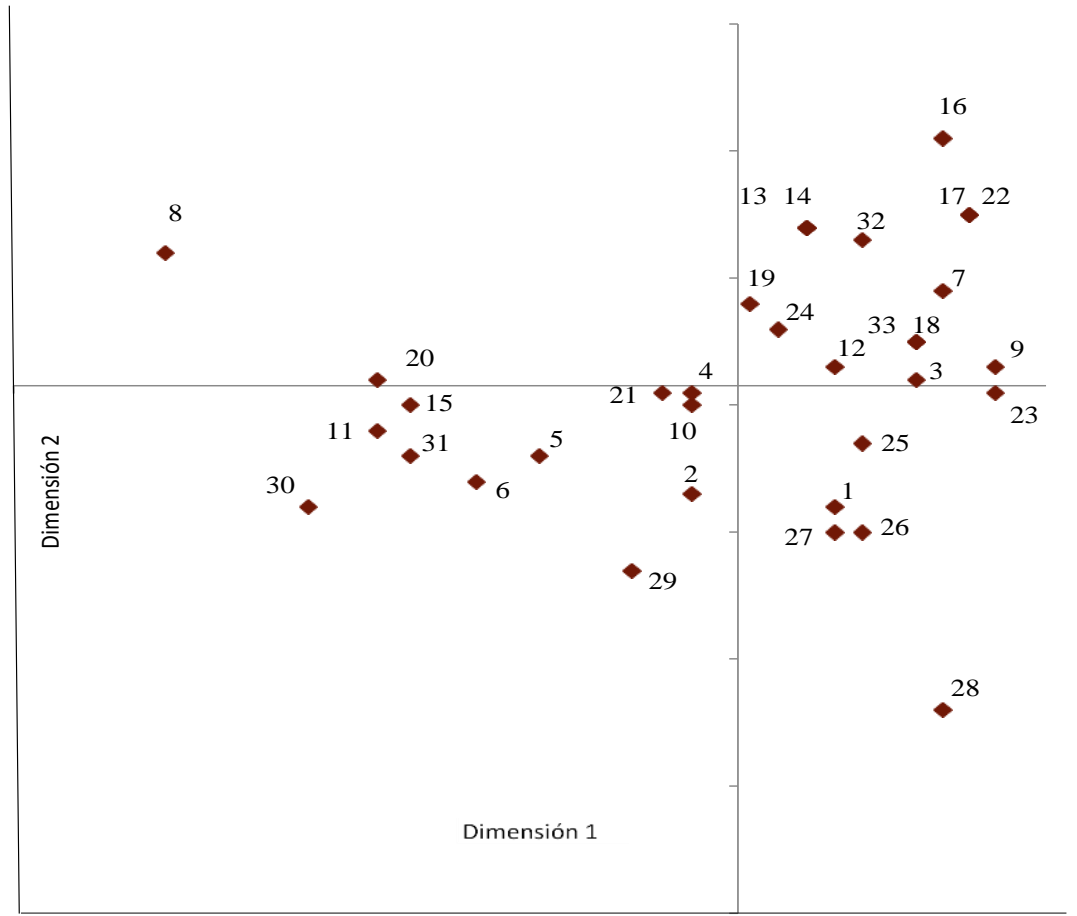

Figura 1. Mapa perceptual

De acuerdo con el mapa de percepción, figura 1, se observa que el cuadrante dos tiene los ítems más significantes de la satisfacción, los cuales señalan los resultados siguientes:

De acuerdo con la pregunta tres ¿qué tan importante y satisfecho estás en que la UPFIM se hagan más prácticas en lugar de teoría?, y de acuerdo al mapa perceptual se tiene una importancia y satisfacción alta.

En consecuencia, en la pregunta 12 ¿qué tan importante y satisfecho estás en que los administrativos sean amables y orientan en los trámites que se requieran (servicio social)? Así mismo se ve la importancia y la satisfacción de los alumnos para que el personal sea amable.

Así mismo en la pregunta 24 se realiza ¿Qué tan importante y satisfecho estarías si la Institución cumple con tus expectativas?, y está ubicado en el cuadrante de mayor importancia y satisfacción alta por lo que sí es importante que se cumplan con lo que promete la universidad para que genere credibilidad ante la sociedad.

De acuerdo con la pregunta 19 ¿qué tan importante y que tanto te satisface los congresos y simposium de las diferentes carreras? por lo que es otro indicador en el segundo cuadrante con una importancia y satisfacción alta.

Otro de los indicadores de acuerdo al cuadrante dos es la número 33 ¿qué tan importante y satisfecho estás con el servicio recibido por la UPFIM en cuanto la biblioteca actual que se tiene?, donde para los educando si tiene mucha importancia la biblioteca actual, así como también están satisfechos.

Así mismo la pregunta 18 ¿qué tan importante y que tan satisfecho consideras en involucramiento con los investigadores en proyectos que ayudan a la sociedad para ir aprendiendo?, de acuerdo al cuadrante esta variable tiene una satisfacción alta y una importancia alta para los educandos, por lo que los investigadores tienen que integrar a los alumnos en proyectos de investigación. 
Otra de las preguntas fue la nueve ¿qué tan importante y satisfecho estás con el servicio recibido por la UPFIM en cuanto al equipo adecuado para prácticas de laboratorio y material que se requiera?, por lo que se concluye que la universidad tiene que contar con equipo adecuado para prácticas de laboratorio y el material requerido por parte de los educandos.

En cuanto a la pregunta siete ¿qué tan importante y que tan satisfecho consideras que el Plan de la carrera está acorde a las necesidades del mercado laboral?, por lo que este indicador se encuentra en el cuadrante dos superior en que tiene una importancia y satisfacción alta, por lo que es importante que las instituciones de educación superior tomen en consideración el mercado laboral al momento de abrir carreras.

Otro indicador que tiene mayor importancia y satisfacción es lo que se pregunta en la 32 ¿qué tan importante es que la UPFIM haya áreas verdes?, este indicador se encuentra en el cuadrante dos de mayor importancia y satisfacción por lo que de acuerdo al mapa de percepción se debe de tener áreas verdes la universidad ya que es una cualidad que les interesa a los educandos.

Así mismo se procedió a la pregunta 14 ¿qué tan importante y que tan satisfecho te sientes que en la UPFIM el profesor motive a los alumnos a continuar sus estudios?, se puede observar que esta variable se encuentra en el segundo cuadrante del mapa perceptual lo que indica que los alumnos deben de motivar constantemente a los alumnos.

Otra de las preguntas de mayor impacto en esta investigación es la 13. ¿qué tan importante y que tan satisfecho te siente en que la universidad te brinde plan de estudios reconocidos por la SEP?, esta variable es de suma importancia ya que deben de estar registrados y reconocidos debido a que esto genera confianza para los educandos.

Bajo este tenor se procedió a realizar la pregunta 17 ¿qué tan importante y que tan satisfecho te sientes en la Universidad si los profesores se capacitan y actualizan constantemente?, esta es una de las variables muy positivas en toda institución ya que la capacitación y actualización constante de los docentes contribuye al mejoramiento de la misma, por lo que esta variable de acuerdo al mapa perceptual se encuentra en el cuadrante importancia alta y satisfacción alta.

Así mismo en la pregunta 22 ¿qué tan importante y satisfecho te siente que la universidad cuente con atención del servicio médico y medicamentos dentro de la institución?, una variable que hay que poner mucha atención es atención de servicio médico y suficiencia en medicamentos para atender a los educandos en cuestión de salud por lo que también se encuentra en el cuadrante dos del mapa perceptual que indica una importancia alta y satisfacción alta por parte de los estudiantes.

Por último, se puede observar en el cuadrante, dos superior, del mapa perceptual donde indica importancia y satisfacción alta es la pregunta 16. ¿qué tan importante y satisfacción sientes que la universidad cuente con profesores con nivel de postgrado? Esta variable juega mayor relevancia para los alumnos de la universidad debido a que se considera que mientras más grados tiene un maestro tienen una mejor percepción de acuerdo al aprendizaje de los educandos y hay que ponerle mayor importancia.

\section{Discusión}

Partiendo de los resultados, se considera que para que los educando sientan satisfacción en la institución, tienen que aplicar estrategias de marketing educativo relacionado a lo que demandan los estudiantes, esto concuerda con la hipótesis general del estudio que indica las estrategias de marketing educativo permiten la satisfacción de los alumnos, considerando que es una hipótesis descriptiva por lo que se analizó a través de frecuencia con el mapa perceptual y es por ello que 
se hace de utilidad esta investigación porque a partir de la percepción de la importancia y satisfacción se pueden establecer estrategias para la satisfacción de los universitarios.

Si bien es cierto como menciona Manes (2015), el marketing educativo es un proceso de investigación, es decir que tiene que pasar por varias etapas, que permitan satisfacer las necesidades de los educandos, es decir desarrollar servicios educativos tendientes a satisfacerlas, acorde al valor percibido, distribuido en tiempo y espacio.

Con base a los resultados señalados con anterioridad a través del mapa perceptual existe evidencia para contestar la pregunta de investigación en relación a : ¿Cuáles son las estrategias del Marketing educativo que permitan la satisfacción de los alumnos de la Universidad Politécnica de Francisco I. Madero?, es decir de todos los que se propusieron en el instrumento de investigación se tiene que dar mayor importancia a las que quedaron cerca de las coordenadas del cuadrante mencionado anteriormente. Por lo que se logra identificar a través de esos indicadores las siguientes estrategias que permitirán la satisfacción de los educandos, de acuerdo con el contraste realizado en la estadística.

Las estrategias de marketing educativo que se tienen que integrar para la satisfacción de los alumnos son:

- Mostrar al alumno un listado de empresas donde puedan aplicar su servicio social o estancia, en caso de que el alumno quiera hacerla en una empresa fuera del listado se le pedirá que proporcione los teléfonos del posible contacto y una persona del departamento de estancias y estadías se pondrá en contacto para ayudar al alumno a obtener el permiso.

- Ampliar los puntos de acceso de la red inalámbrica universitaria para lo cual se tiene que planear zonas donde se pongan mesitas para que los estudiantes puedan tener acceso a la conexión.

- Hacer convenios de intercambio con universidades extranjeras que tengan carreras similares a las que se ofertan en la UPFIM como: Argentina, Cuba, España, América del Norte y Canadá.

- Asignar a los interesados un investigador para orientarlo en el proyecto a realizar en caso de que quiera hacer intercambio fuera del país, el área de vinculación ayudará al interesado en el trámite y llenado de formatos, así como en la comunicación de enlace con la persona de vinculación de la otra universidad para que sea expedida y recibida la autorización por parte del director del programa educativo.

- En la página de la universidad se encuentren publicaciones elaboradas por académicos de la misma, en donde se da a conocer acerca de determinados temas.

- Diseñar resumen de lo más importante de las publicaciones donde se mostrará el contenido, es decir el know how, la metodología o pasos de las investigaciones con los principales resultados.

- Se revisarán los expedientes de los alumnos que tienen este tipo de beca en la cual al hacer estudio socioeconómico y verificar que su tutor o padre de familia gane dos salarios mínimos, (menor de $\$ 2,562.00$ mensuales) de lo contrario quitarles ese apoyo a los alumnos que no lo necesiten.

- Revisar los expedientes de alumnos e identificar aquellos que tengan alguna discapacidad motriz, visual o auditiva, así como alumnas que estén embarazadas para incluirlas en la beca.

- Difundir en la página web los proyectos de los profesores investigadores, así como su CVU, para que los interesados en algún tema puedan contactar al investigador y consultar sus preguntas.

- Crear una página en Facebook donde se informe de proyectos efectuados por la UPFIM.

- Solicitar en el área de tutorías la materia que se desea para formar un grupo con los solicitantes y asignar un docente que apoye en temas especiales o unidades. 
- Por medio de un correo solicitar apoyo en temas donde se tiene problemas de comprensión a la encargada de tutorías para definir horario de ayuda por un docente el cual puede ser el mismo que da la asignatura u otro que tenga el perfil en esas materias.

- Crear una cuenta para entrar a la biblioteca digital ECEST.

- Brindar acceso al centro de cómputo para consultar libros digitales, o en su caso en algún otro lugar donde tengan acceso a internet.

- Proponer crear un sitio web donde pueda bajar material (audio, videos) para aprender o repasar el idioma inglés.

- Poner a disposición del público en general recursos de forma intelectual y asesoría en análisis de suelos, producción animal.

- Asesorar a microempresarios interesados en la planeación, así como indicarle los programas sociales y dependencias donde puede solicitar financiamiento con apoyo de los alumnos.

\section{Referencias}

Alvarado, O. (2003). Gerencia y Marketing Educativo. Lima: Universidad Alas Peruanas.

Cadena-Badilla, M., Mejías, A., Vega, A., \& Vázquez, J. (2015). La satisfacción estudiantil universitaria: análsiis estratégico a partir del análisis de factores. Industrial Data, 18(1), 9-18. Recuperado de https://www.redalyc.org/pdf/816/81642256002.pdf

Carneiro, M. (2010). Dirección Estratégica Innovadora. (M. Martínez, Ed.) España: Netbiblo.

Chandler, A. (2003). Strategy and Structure. Chapters in the history of the American Industrial Enterprise. New York: Beard Books.

Elliott, K. M., \& Healy, M. A. (2001). Key factors influencing student satisfaction related to recruitment and retention. Journal of marketing for higher education, 10(4), 1-11.

Espinoza, C. (2010). Metodología de investigación tecnológica. Pensando en sistemas. Ira edición. Huancayo: Imagen Gráfica.

Garbanzo, G. (2007). Factores asociados al rendimiento académico en estudiantes universitarios, una reflexión desde la calidad de la educación superior pública. Revista Educación, 31(1), 43-63.

Gobierno del Estado de Hidalgo. (2016). Plan Estatal de Desarrollo Hidalgo Programas especiales. Programa Institucional de Desarrollo de la Universidad Politécnica de Francisco I. Madero. 2017-2022. Hidalgo.

Hernández, R., Fernández, C., \& Baptista, P. (2014). Metodología de la Investigación. México: McGraw-Hill.

Hill, N., Brierley, J., \& MacDougall, R. (2003). How to measure customer satisfaction. Inglaterra: Gower Publishing Limited.

Jiménez, G., Terriquez, C., \& Robles, Z. (2012). Evaluación de la satisfacción académica de los estudiantes de la Universidad Autónoma de Nayarit. Revista Fuente, 3(6), 46-56. Recuperado de http://fuente.uan.edu.mx/publicaciones/02-06/8.pdf

Lago, D., López, E., Municio, P., Ospina, R., \& Vergara, G. (2013). La Calidad de la Educación Superior. ¿Un reto o una utopía? Bogotá: Ecoe.

Larios-Gómez, E. (2014). Mercadotecnia de las instituciones de educación superior. Cuaderno Profesional de Marketing-UNIMEP, 2(1), 15-29.

Manes, J. (2004). Marketing para Instituciones Educativas: Guía para planificar la captación y retención de alumnos. Buenos Aires: Granica.

Manes, F. (2015). ¿Qué le hace la música a nuestro cerebro?. El País.

Salinas, A., \& Martínez, P. (2007). Principales factores de satisfacción entre los estudiantes universitarios. La Unidad Académica Multidiciplinaria de Agronomía y Ciencias de la UAT. Revista Internacional de Ciencias Sociales y Humanidades, SOCIOTAM, XVII(1), 163-192. Recuperado de https://www.redalyc.org/pdf/654/65417108.pdf

Sánchez, J. (2018). Satisfacción estudiantil en educación superior: validez de su medición. Santa Martha: Universidad Sergio Arboleda. 
Sinclaire, J. (2014). An empirical investigation of student satisfaction with college courses. Research in Higher Education Journal, 22, 1-21. Recuperado de https://eric.ed.gov/?id=EJ1064140

Spreng, R., MacKenzie, S., \& Olshavsky, R. (1996). A Reexamination of the Determinants of Consumer Satisfaction. Journal of Marketing, 60(3), 15-32.

Zapata, E. (2012). Mercadeo Educativo. Estrategias para promover instituciones y programas. Bogotá: Hipertexto SAS. 